\title{
L'Émergence de Voix Distinctes: pratiques singulières de l'art dramatique à l'école et dans la communauté
}

\author{
Carole Marceau \\ Maud Gendron-Langevin \\ Université du Québec à Montréal - Montréal, Canada
}

RÉSUMÉ - L'Émergence de Voix Distinctes: pratiques singulières de l'art dramatique à l'école et dans la communauté - Dans le cadre de cet article, la question des formations singulières offertes dans le milieu scolaire et communautaire est abordée. Au Québec, la formation artistique fait partie du programme de formation de l'École québécoise. Pourtant, certains milieux occupent des créneaux spécifiques en permettant à des jeunes de prendre la parole dans un contexte particulier. Par le biais d'un question naire, un tableau comparatif est établi afin de dégager les éléments convergents et divergents des deux groupes. Finalement, des recommandations et des pistes de recherche sont mises de lavant afin d'étendre cette étude à un plus grand nombre d'écoles et d'organismes communautaires.

Mots-clés: Formation en Art. Pédagogie. Art Dramatique. Organisme Communautaire. Adolescents Marginalisés.

ABSTRACT - The Emergence of Distinctive Voices: unique practices of drama at school and in the community - This article will discuss the unique education programs provided in school and in community-based settings. Arts education is part of the Quebec Education Program, but certain environments offer specific activities giving youth the opportunity to express themselves in a particular context. Following responses to a questionnaire, a comparative chart was created, putting forth the differences and similarities amongst those groups. Finally, recommendations and future research ideas were formulated, in order to extend this study to a wider number of schools and community centers in the province of Quebec.

Keywords: Arts Education. Pedagogy. Drama. Community Organization. Marginalized Adolescents.

RESUMO - A Emergência de Vozes Distintas na Escola e na Comunidade: práticas singulares de ensino de teatro no Quebec - Neste artigo, aborda-se a questão dos cursos específicos propostos em dois grupos: no ambiente escolar e no ambiente comunitário. Neste caso, a formação artística faz parte do programa de formação da escola do Quebec. Entretanto, algumas organizaçóes realizam atividades específicas, permitindo que jovens assumam a palavra em um contexto particular. Por meio de um questionário, foi realizado um quadro comparativo para identificar elementos convergentes e divergentes dos dois grupos. Finalmente, foram formuladas indicaçóes e pistas de pesquisa com o objetivo de fazer com que este estudo atinja um maior número de escolas e de organizaçóes comunitárias. Palavras-chave: Formaçáo em Arte. Pedagogia. Pedagogia do Teatro. Organizaçáo Comunitária. Adolescentes Marginalizados. 
L'art dramatique et le théâtre en milieu scolaire et communautaire se pratiquent de plusieurs façons à travers le monde. Au Québec, certaines écoles et certains organismes développent néanmoins des programmes singuliers. Ceux-ci permettent une expression et une exploration du médium du théâtre à des fins qui dépassent ce qui est prescrit par le Programme de formation de l'école québécoise et le simple loisir. Ayant nous-mêmes enseigné au secondaire et travaillé dans le milieu communautaire pendant de nombreuses années, notre expérience du terrain a laissé place au désir de porter un regard critique sur ces pratiques distinctives. Le point de départ de cette recherche exploratoire est donc issu de la pratique, et se veut une étude de cas répondant à quelques questions permettant de mettre en lumière les caractéristiques propres à ces formations: ce qui les distingue, mais aussi, ce qui les unit. Nous nous sommes demandé si le milieu scolaire et le milieu communautaire poursuivaient des objectifs communs et faisaient face à des obstacles semblables malgré leur contexte, leur clientèle et leur offre de services complètement différents. Nos hypothèses de départ ont été de deux ordres: a) pour les projets particuliers de formation, l'art dramatique est une fin en soi (connaissances historiques, maîtrise des techniques théâtrales) - par exemple, une formation esthétique et artistique rigoureuse peut, entre autres, amener les élèves à être prêts pour les formations professionnelles postsecondaires en théâtre; b) les organismes communautaires faisant appel à l'art dramatique utilisent ce langage artistique comme moyen pour l'atteinte d'objectifs sur le plan du développement personnel et social des participants (habiletés sociales, connaissance de soi et développement de l'estime de soi). Par exemple, peu importe le résultat esthétique final, l'objectif est de faire vivre un processus permettant une préparation à la vie citoyenne.

Pour tenter de confirmer ou d'infirmer ces hypothèses, nous nous intéresserons à différents types de projets s'adressant à des clientèles particulières dans la grande région métropolitaine de Montréal au Québec. Nous tenterons ainsi de faire un premier portrait ciblé d'organismes communautaires et de projets particuliers de formation en faisant ressortir les points communs entre ces pratiques et les liens qui unissent les pratiques en milieu scolaire et communautaire. Enfin, puisque cela n'a pas encore été fait au Québec, malgré une vie culturelle foisonnante, cette étape nous permettra de poser les premiers jalons d'une recherche à plus grande échelle qui nous 
donnerait l'occasion de dresser un portrait plus juste des pratiques singulières au Québec, des causes et caractéristiques qui déterminent leurs réalités diverses et des impacts de ces pratiques sur les participants et dans les communautés où elles se situent.

En ce qui concerne notre cadre méthodologique, nous préciserons quels sont les participants ainsi que les principales stratégies de collecte de données. Notre recherche exploratoire est une étude de cas. Celle-ci dressera un premier portrait des formations pratiques singulières de l'art dramatique dans la grande région métropolitaine de Montréal et dégagera des pistes de réflexion qui serviront d'amorce à une éventuelle recherche. Tel que nous l'avons mentionné précédemment, nous aimerions éventuellement étendre cette étude à un plus grand nombre d'écoles et d'organismes communautaires au Québec.

Dans un premier temps, nous avons recensé les différentes écoles et organismes communautaires qui offrent des formations particulières en art dramatique. Au Québec, il existe plusieurs programmes particuliers de formation en art (art dramatique, arts plastiques, danse et musique), tant au primaire qu'au secondaire. Nous avons cependant choisi de circonscrire notre recherche en sélectionnant quatre écoles secondaires et trois organismes communautaires qui répondaient à nos critères de recherche soit: une formation singulière en art dramatique, située dans la grande région métropolitaine de Montréal et s'adressant à des jeunes de douze à dix-sept ans.

Par le biais d'un questionnaire, nous avons procédé à une collecte de données qui cherche à mieux connaître et comprendre les objectifs liés à ces programmes particuliers qui s’adressent à un même groupe d'âge, mais qui œuvrent dans des contextes différents. En posant les mêmes questions aux deux groupes, nous pensons être en mesure de relever d'une part les différences et les similitudes reliées aux objectifs des programmes et des pratiques qui s'y rattachent ainsi que les obstacles à leur atteinte; d'autre part, nous estimons pouvoir identifier les retombées possibles sur le développement individuel et collectif du jeune et de son milieu de vie. Voici les questions posées aux deux groupes': 1) qu'est-ce qui a donné lieu ou a motivé la mise sur pied de votre programme? 2) veuillez nous décrire sommairement les particularités de votre programme: a) objectifs (mission, mandat); b) clientèle (groupe d'âge, milieu socioéconomique); c) type de formation offerte (courtes descriptions, durée etc.); d) depuis combien de temps ce programme est-il offert? 3) qu'est-ce que cette formation 
apporte aux participants à court terme et à long terme? 4) quelles sont les retombées plus larges dans le milieu (communauté)? 5) quelles sont les difficultés rencontrées depuis la mise sur pied de votre programme (acceptation dans le milieu, budget, recrutement etc.)?

Nous avons codifié les données recueillies en les organisant et les triant en catégories, thèmes et sous-thèmes, dans le but de faciliter leur analyse. Par la suite, nous avons mis en exergue les objectifs et retombées communs entre les deux groupes ainsi que les difficultés vécues tant pour les programmes particuliers de formation dans les écoles que pour les organismes communautaires ouvrant dans les différents quartiers de la ville de Montréal.

Cette recherche exploratoire comporte certaines limites, notamment en ce qui concerne l'échantillon restreint et le territoire circonscrit. Ces limites ont été établies à des fins de réalisme en termes de temps et de terrain. Aussi, la nature des questions posées ne permet pas de fournir de plus amples résultats concernant l'impact des pratiques en question sur les participants (ex.: résultats scolaires) et leur milieu (ex.: meilleure intégration sociale). Pour atteindre ces objectifs, une recherche ultérieure nécessiterait une collecte de données auprès des participants ${ }^{2}$, de leurs familles, des intervenants et des représentants de la communauté où les activités sont implantées.

\section{L'Art Dramatique à l'École Québécoise}

Lactuel programme en art dramatique fait partie du cursus scolaire des élèves du primaire et du secondaire. Depuis le renouveau pédagogique $^{3}$ (2001-2006) cette discipline artistique - tout comme les arts plastiques, la danse et la musique - fait partie du domaine des arts, l'un des six domaines d'apprentissage du Programme de Formation de l'École Québécoise. Ces disciplines artistiques ont toutes la même visée, soit d'amener l'élève à «[...] transposer, à l'aide d'un langage symbolique, sa représentation du réel et sa vision du monde dans des situations de création, d'interprétation et d'appréciation variées» (Ministère de l'Éducation, du Loisir et du Sport, 2007, p. 3).

Au Québec les arts sont obligatoires à l'école. Cependant, la grille-matière, élaborée par le directeur, les enseignants et le conseil d'établissement ${ }^{4}$, détermine le nombre de minutes d'enseignement pour chaque matière et s'assure d'allouer suffisamment de temps pour couvrir correctement le programme à l'étude. 

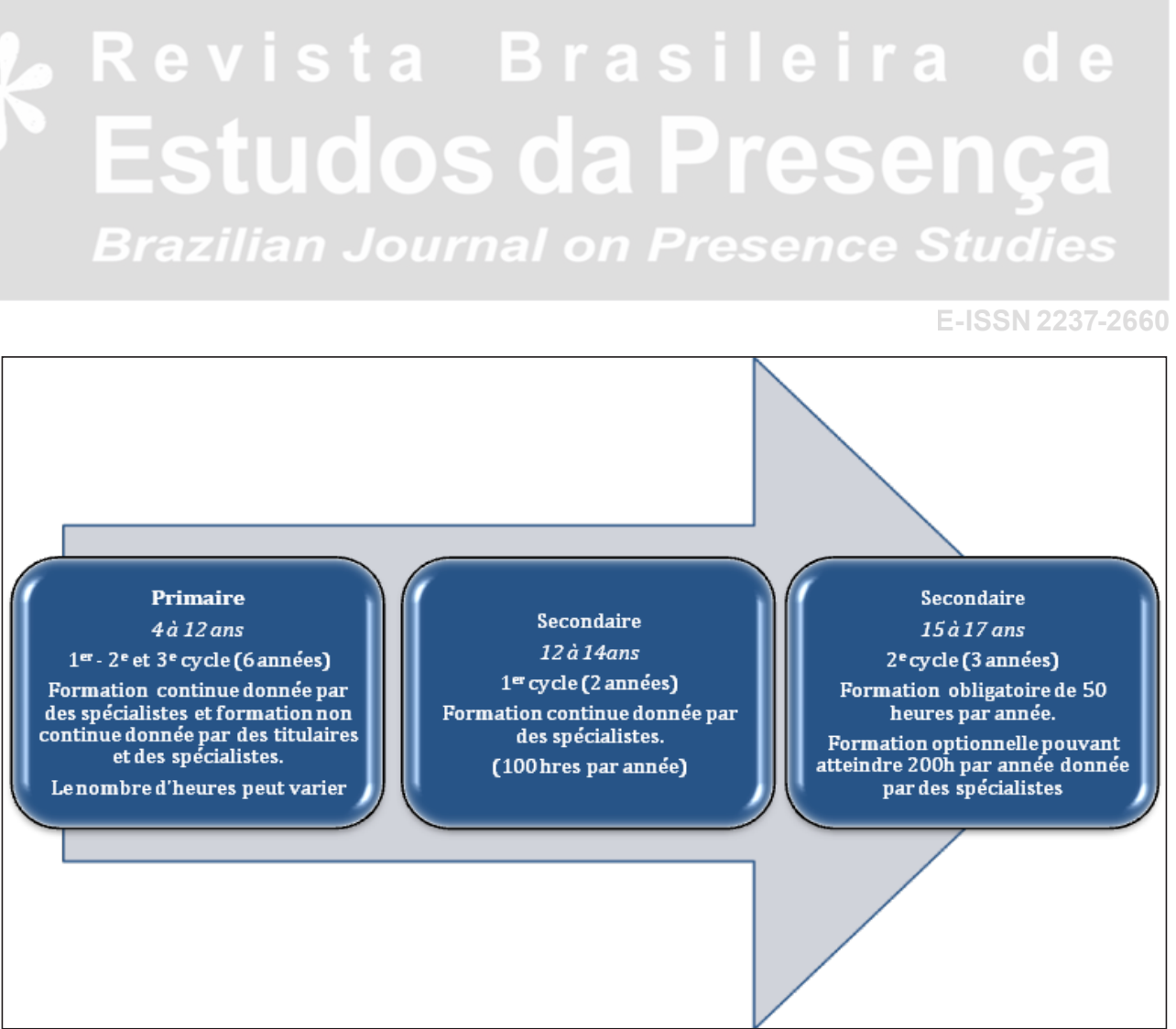

Image 1 - Tableau sur l'organisation de l'offre de cours en art au primaire et au secondaire.

Au primaire, le régime pédagogique prévoit l'enseignement d'un minimum de deux disciplines artistiques choisies par l'école. La première est enseignée en continuité de la première à la sixième année tandis que la deuxième n'a pas cette contrainte et peut être modifiée année après année. Cette deuxième discipline est très souvent enseignée par le titulaire ${ }^{5}$ de la classe, même si les associations des enseignants ainsi que les syndicats revendiquent l'engagement de spécialistes ${ }^{6}$ en enseignement des arts.

$\mathrm{Au}$ secondaire, la formation artistique est plus diversifiée. Durant le premier cycle, les écoles sont tenues d'offrir un minimum de deux des quatre disciplines artistiques sur deux années consécutives. Au deuxième cycle, l'élève fait de nouveau le choix d'une discipline artistique en continuité ou non, jusqu'à la fin de son secondaire. Cependant, peu importe la discipline choisie, il doit réussir son cours d'art à la quatrième secondaire s'il veut obtenir son diplôme d'études secondaires. Les écoles désirant offrir une formation plus poussée dans l'une ou l'autre des disciplines artistiques peuvent décider d'augmenter le nombre d'heures allouées à cette formation obligatoire en proposant un projet particulier en art.

\section{Les Caractéristiques des Projets Particuliers ${ }^{7}$ de Formation en Arts}

Les projets particuliers font partie de la formation optionnelle. Ils désignent ce que l'on connaît généralement sous les appellations concentration en arts et projet arts-études. Ils visent à enrichir la for- 
mation de base de l'élève à partir d'une ou de plusieurs disciplines artistiques présentes dans le Programme de formation de l'école québécoise (art dramatique, arts plastiques, danse et musique).

Les projets particuliers, en étroite relation avec la mission et le projet éducatif de l'école, résultent de la volonté de donner aux élèves une formation distinctive en arts qui leur permet de se développer harmonieusement et, le cas échéant, de se préparer à des études supérieures en arts. Certains de ces programmes sélectionnent les élèves sur la base des résultats scolaires ou de certaines aptitudes et attitudes jugées indispensables à la formation, tandis que d'autres n'exigent qu'un intérêt marqué pour la discipline artistique qui sera développée.

Ce programme de cinq unités et plus doit être approuvé par la ministre puisqu'il déroge à la grille-matière prévue au régime pédagogique. Il s'applique à un groupe d'élèves ou à une école en vertu de la loi sur l'instruction publique ${ }^{8}$. Ces formations requièrent plus de temps, elles ont donc un impact sur l'organisation interne de l'école. La fédération autonome de l'enseignement ${ }^{9}$ (FAE) s'est penchée sur les retombées de ce type de formation tant pour la qualité de la formation que pour le contexte d'enseignement (Fédération Autonome de L'Enseignement, 2012).

Les retombées dans le milieu scolaire selon la Fédération autonome de l'enseignement (FAE)

\begin{tabular}{|l|l|}
\hline \multicolumn{1}{|c|}{ Positifs } & \multicolumn{1}{c|}{ Négatifs } \\
\hline $\begin{array}{l}\text { Une formation générale améliorée; } \\
\text { La reconnaissance et la promotion } \\
\text { de champs d'intérêts dans divers } \\
\text { domaines de formation; }\end{array}$ & $\begin{array}{l}\text { Ne respecte pas toujours le prin- } \\
\text { cipe de l'égalité de chances; }\end{array}$ \\
* Éclatement de la formation conti- \\
nue;
\end{tabular}

Image 2 - Tableau des retombées des projets particuliers de formation selon la FAE (2012). 
À la lumière de ces données, nous remarquons que ce syndicat (FAE) s'intéresse plus particulièrement aux aspects qui touchent la tâche des enseignants et le contexte particulier de cet enseignement. En effet, il semblerait que les enseignants impliqués dans ces projets le font de manière volontaire, ce qui aurait un effet positif puisqu'on reconnaît par ce fait même leurs champs d'intérêt et leur discipline. En contrepartie, certains dénoncent l'iniquité dans la tâche d'enseignement, ce qui aura sans doute un impact sur les difficultés rencontrées pour maintenir ces projets viables. Fait surprenant, on y retrouve très peu d'éléments se rapportant au développement de l'élève sur le plan disciplinaire, individuel et social. Par contre, on remarque que ces projets attirent et interpellent les parents intéressés par les écoles se démarquant par une formation plus poussée. Il sera intéressant, lors de la présentation des écoles à vocation artistique, de faire ressortir les retombées positives et négatives de cette formation sur le développement individuel et social de l'élève ainsi que sur son milieu de vie.

Parallèlement au milieu scolaire, le réseau communautaire offre aussi des activités de loisir permettant d'enrichir les connaissances des élèves et de leur faire vivre des expériences artistiques variées. De fait, nombre de centres culturels et d'organismes communautaires au Québec proposent des formations et des projets spécifiques aux arts tout en s'adressant à des clientèles particulières. Les sections qui suivent présenteront d'abord les politiques culturelles liées aux organismes communautaires pour ensuite faire le pont entre le milieu scolaire et communautaire.

\section{Les Politiques Culturelles Liées aux Organismes Communautaires}

Le Ministère de la Culture, des Communications et de la Condition Féminine (MCCCF) est responsable, au Québec, des politiques culturelles en matière de loisirs. De manière quinquennale et depuis de nombreuses années, le ministère fait une enquête sur les pratiques culturelles au Québec ${ }^{10}$ afin de mieux en connaître les caractéristiques et ainsi, de mieux servir la population. La dernière étude a été publiée en 2012 et porte sur des statistiques issues de $2009^{11}$. Voici un tableau synthétisant une sélection de données pertinentes pour cet article. 


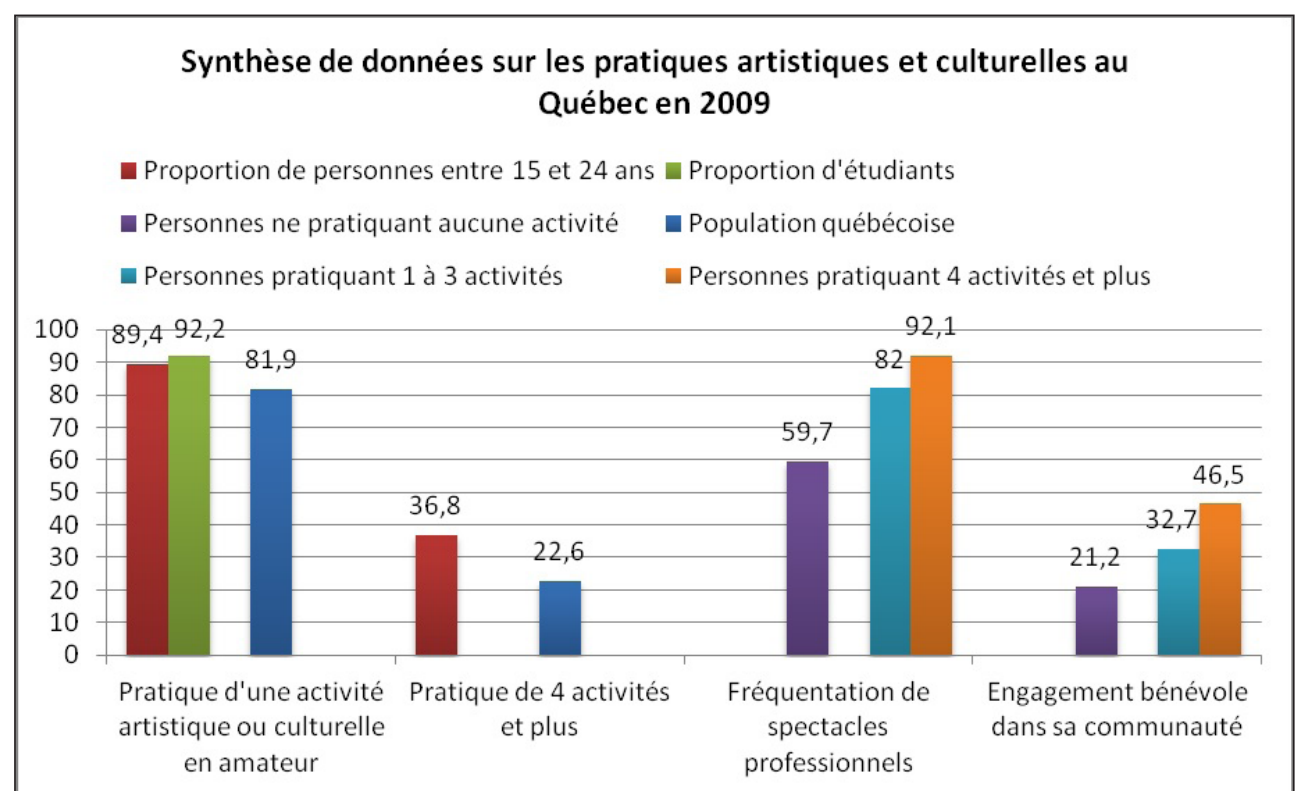

Image 3 - Tableau synthèse tiré d'une sélection de données de la septième Enquête sur les Pratiques Culturelles au Québec (2012).

L’enquête révèle que $81,9 \%$ de la population québécoise s'adonne à des activités artistiques ou culturelles en amateur ${ }^{12}$ et que $89,4 \%$ de ceux-ci sont âgés de quinze à vingt-quatre ans. Parmi cette tranche d'âge, c'est la population étudiante qui obtient le taux de participation le plus élevé. Les quinze à vingt-quatre ans sont aussi plus nombreux à s'adonner à plusieurs activités de loisir culturel, avec 36,8 \% pratiquant 4 activités et plus. L’enquête énonce que la participation décroît avec l'âge, mais augmente avec le revenu du ménage et le niveau de scolarité. En outre, l'enquête fait état de liens entre la pratique artistique et culturelle et la fréquentation de spectacles selon le nombre d'activités exercées. Il en va de même pour le bénévolat avec 46,5\% pour ceux pratiquant quatre activités et plus, contre $32,7 \%$ pour ceux en faisant un à trois et $21,2 \%$ pour ceux qui n'en pratiquent pas. Enfin, l'enquête soutient que la pratique libre d'activités artistiques chez les quinze à vingt-quatre ans est de $89,4 \%$ contre $25,1 \%$ pour les activités artistiques organisées ${ }^{13}$.

Nul doute que cette enquête démontre l'importance des pratiques culturelles et artistiques en amateur, c'est-à-dire dans le secteur des loisirs (organismes communautaires, activités parascolaires), chez les jeunes d'âge scolaire. Nous pouvons aussi supposer que la pratique artistique et culturelle contribue au développement des publics et à l'engagement citoyen. Il n'est pas surprenant, dès lors, de constater la multiplication des activités de ce secteur tant à l'école que dans le milieu communautaire. 
Afin de mieux comprendre le fonctionnement et les valeurs qui sous-tendent le milieu communautaire, il importe de donner quelques définitions. Par loisir communautaire, la Fédération québécoise des centres communautaires entend qu'il s'agit d'une:

[...] approche du FAIRE AVEC [qui] favorise la reconnaissance des forces de chacun, renforce le potentiel des gens, permet de créer un milieu de vie et d'accroître le sentiment d'appartenance [...]. Le loisir est qualifié de communautaire en raison de l'objectif de contribution au développement social qui traverse l'action des centres [communautaires] dans leur milieu (Fédération Québécoise des Centres Communautaires, 2013, p. 4).

Il y a donc, dans la vision communautaire, un aspect lié au développement social et citoyen qui correspond étroitement aux constats de l'étude du MCCCF concernant l'engagement civique. De fait, l'implication des participants dans des activités culturelles et artistiques offertes dans leur quartier crée un sentiment d'appartenance au milieu et contribue à l'essor et au développement de celuici. L'Association Québécoise du Loisir Municipal va dans le même sens en affirmant que l'objectif fondamental de l'offre de service, c'est-à-dire «[...] la correspondance [entre les activités et] les besoins identifiés et la réduction des contraintes de tout ordre pouvant nuire à l'accessibilité au loisir», est davantage assuré lorsqu'il y a engagement citoyen (Association Québécoise du Loisir Municipal, 2002, p. 8).

En ce sens, il est intéressant de constater que l'accessibilité aux loisirs est l'affaire de tous, c'est-à-dire de tous les citoyens, quels que soient leur âge, leur statut socioéconomique, leur provenance culturelle et leurs capacités physiques et mentales. En effet, les politiques culturelles du Québec et les mandats des différents organismes dédiés à la promotion et la pratique des loisirs tiennent compte des caractéristiques de personnes susceptibles de s'impliquer dans des loisirs artistiques et culturels. L'Institut de la Statistique du Québec a déposé un rapport en 2010 sur la participation et les limitations aux activités de personnes en situation de handicap entre 2001 et 2006. On y indique entre autres que les personnes de 15 ans et plus en situation de handicap participent à des activités de loisir à l'extérieur de leur résidence dans une proportion de 46,4\%. On apprend aussi que 27,8 \% d'entre eux font du bénévolat. Ces chiffres démontrent que la demande en loisir est bien présente chez cette clientèle. L'étude ajoute que celle-ci pourrait être plus élevée si l'accessibilité et 
l'adaptation des loisirs étaient mieux planifiées et organisées dans les municipalités. L'Association Québécoise du Loisir Municipal (2002), dans un rapport sur les loisirs auprès de clientèles ayant des besoins particuliers, inclut non seulement les personnes ayant une déficience intellectuelle, mais aussi les membres de communautés culturelles dans sa réflexion sur la qualité des services de loisirs offerts et les problématiques rencontrées. On y fait état de similitudes dans les réalités de ces deux groupes distincts. Celles-ci concernent les situations de discrimination et d'exclusion vécues, dues au fait que les services ne soient pas toujours adaptés à leur situation. Les recommandations issues de cette réflexion sont de l'ordre de l'intégration sociale et culturelle, de la participation citoyenne dans sa communauté et de la reconnaissance des spécificités et des besoins de ces clientèles.

\section{La Jonction entre le Communautaire et le Scolaire}

Les politiques culturelles du Québec tiennent comptent à la fois des secteurs professionnel, scolaire et communautaire. Ces secteurs sont étroitement liés par le rôle qu'ils jouent dans la diffusion, la réception et la pratique des arts. Le Ministère de la Culture, des Communications et de la Condition Féminine a donc pour mandat de soutenir et encourager les arts, notamment en "[favorisant] le développement du loisir culturel [en promouvant] sa pratique et [en en facilitant] l'accès» (Ministère de la Culture, des Communications et de la Condition Féminine, 2013, n.p.). En 1992, la politique du gouvernement du Québec en matière de culture affirmait même que «[...] l'État a le devoir de soutenir et de développer la dimension culturelle de la société avec une vigueur au moins comparable à celle qu'il met à soutenir et à promouvoir les dimensions sociales et économiques de cette même société» (Ministère de la Culture, des Communications et de la Condition Féminine, 2013, p. 9) - ceci est un signal fort que la culture, sous toutes ses formes, devrait être une priorité. Parmi les moyens mis en place, notons que des efforts considérables ont été déployés afin de rapprocher le milieu culturel et scolaire ainsi que pour renforcer l'éducation artistique à l'école. Depuis la politique culturelle de 1996, des programmes ont vu le jour, tels qu'Une École Montréalaise pour Tous en 1997 et La Culture à Ecole en 2000. Le premier vise entre autres, par la formation des équipes-écoles et la médiation culturelle, «[...] à soutenir la réussite scolaire, personnelle et sociale des jeunes montréalais issus des mi- 
lieux défavorisés et des milieux défavorisés pluriethniques» (Ministère de la Culture, des Communications et de la Condition Féminine, 2011, n.p.). Ce programme offre une allocation de $20 \$$ par élève aux écoles desservant ces clientèles afin de pouvoir leur faire vivre des expériences enrichissantes et motivantes (rencontre avec un artiste, visite de lieux culturels, participation à des projets spéciaux etc.). Le second programme met à la disposition des écoles un répertoire de ressources culture-éducation dans lequel figurent plus de deux mille artistes et organismes culturels offrant des expériences artistiques aux élèves du primaire et du secondaire. Ce programme encourage la médiation culturelle, la participation active des élèves à divers des projets artistiques, les incite à prendre part à des activités culturelles et à devenir des consommateurs d'art et, enfin, fait la promotion des professions reliées aux arts (Ministère de l'Éducation, du Loisir et du Sport, 2013). Ces partenariats entre le milieu professionnel, communautaire et scolaire s'inscrivent tout à fait dans la mission du Plan Stratégique du Ministère de l'Éducation, du Loisir et du Sport (Ministère de l'Éducation, du Loisir et du Sport, 2009) qui stipule entre autres son intérêt à "[...] favoriser l'accès aux formes les plus élevées du savoir et de la culture à toute personne qui en a la volonté et l'aptitude» (Ministère de l'Éducation, du Loisir et du Sport, 2009, p. 13).

Enfin, il est possible de constater que ces politiques culturelles, tant dans le milieu professionnel, communautaire et scolaire ont pour objectif ultime d'encourager la participation citoyenne à une vie artistique et culturelle riche et variée. La formation des publics et l'incitation à une pratique artistique amateur vont de pair avec les constats du MCCCF quant à la population la plus active sur ce plan, les élèves. De plus, les clientèles ayant des besoins particuliers sont à prendre en compte dans l'offre et l'organisation des services de loisirs municipaux puisqu' ils participent activement à la vie sociale et culturelle des villes. Il a été question, jusqu'à maintenant, des actions et des politiques culturelles communautaires tous arts confondus. Bien entendu, le présent article traite plus spécifiquement de la pratique de l'art dramatique en contexte scolaire et communautaire. Dans la section qui suit, nous présenterons le portrait de quatre écoles secondaires proposant des projets particuliers de formation ainsi que trois organismes communautaires en loisir culturel offrant des ateliers de théâtre. 


\section{L'Émergence de Voix Distinctes à l'École}

Nous comptons douze projets particuliers de formation en art dramatique au Québec ${ }^{14}$. Nous avons retenu quatre d'entre eux se situant dans la grande région métropolitaine. Leur présentation se fera donc au regard des caractéristiques communes et divergentes selon les sous-critères suivants: 1) projet mis sur pied en réponse à une demande du milieu scolaire ou pour offrir un service; 2 ) objectifs; 3) clientèle et types d'activités offertes; 4) apports aux participants et retombées dans le milieu; 5) difficultés rencontrées.

\section{École Secondaire Armand-Corbeil}

L'école secondaire Armand-Corbeil fait partie de la Commission Scolaire des Affluents. Le programme particulier de formation en art dramatique de cette école, en vigueur depuis vingt ans, provient d'une demande du milieu. Cette formation permet à l'élève durant tout son secondaire de développer des compétences en se référant aux vastes repères culturels de la dramaturgie. Cet enrichissement se traduit par une maitrise accrue des techniques de jeu de l'acteur, d'une expérimentation approfondie des techniques théâtrales et d'une initiation aux métiers de la scène. Pour y être admis, les élèves doivent passer des auditions, les critères reposent essentiellement sur l'intérêt de l'élève pour l'art dramatique et sur sa capacité à travailler en équipe et à collaborer. Le programme de cette formation ne vise donc pas le vedettariat, mais plutôt un esprit de groupe. Cela dit, le niveau d'enrichissement est tel qu'il permet d'approfondir les connaissances et le savoir-faire théâtral.

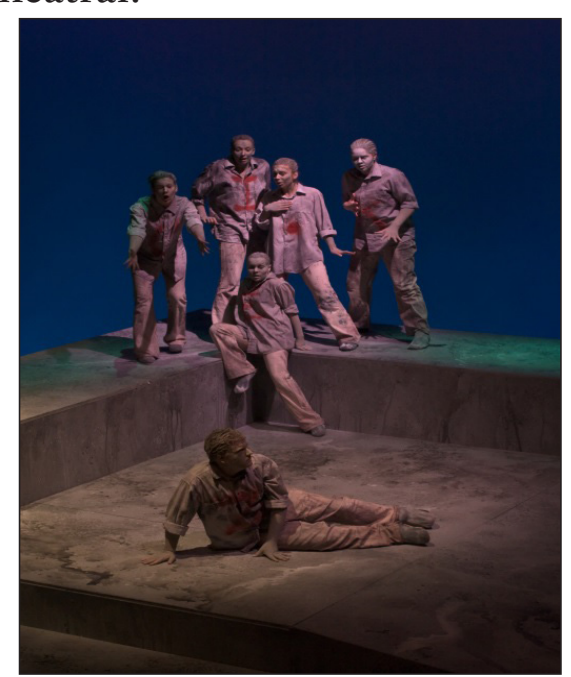

Image 4 -École Armand Corbeil. Texte et mise en scène: Lucie Tremblay. Photo: Véronique Poirrier

Carole Marceau; Maud Gendron-Langevin - L’Émergence de Voix Distinctes: pratiques singulières de l'art dramatique à l'école et dans la communauté Rev. Bras. Estud. Presença, Porto Alegre, v. 5, n. 2, p. 287-312, mai/août 2015. Disponible sur: <http://www.seer.ufrgs.br/presenca> 


\section{École Secondaire Curé-Antoine Labelle}

L'École Curé-Antoine Labelle est une école de quartier faisant partie de la Commission Scolaire de Laval. Les élèves qui la fréquentent proviennent essentiellement des environs, mais elle accueille aussi ceux provenant des autres quartiers qui désirent s'inscrire dans la concentration arts-études. Le désir d'implanter un programme enrichi en art dramatique vient de la commission scolaire, de la volonté d'une enseignante et de l'engagement des élèves quant aux projets d'envergure proposés. Ce sont ces trois éléments qui ont donné lieu, il y a vingt et un ans, à l'envol de ce projet particulier de formation. S'échelonnant sur trois années, les élèves inscrits à cette formation développent des compétences relatives à l'art dramatique de haut niveau, tant pour créer que pour interpréter ou apprécier des œuvres dramatiques. Ce programme permet aux élèves qui y participent de faire de l'art dramatique tous les jours et parfois même deux fois par jour, puisqu'en cinquième secondaire, la formation offre douze cours sur une période de neuf jours. Il n'y a pas d'audition pour suivre cette formation en art dramatique qui admet tous les élèves démontrant de l'intérêt et des aptitudes en art dramatique. Les enseignants spécialistes en art dramatique invitent régulièrement des artistes à partager leur savoir lié au jeu dramatique, à la dramaturgie ou à la théâtralité. Ce qui donne lieu à des créations collectives riches, complexes et variées.

\section{École Secondaire Robert Gravel}

L'École secondaire Robert Gravel est ouverte à tous les jeunes de la grande région montréalaise qui fréquentent le secondaire et qui ont un intérêt marqué pour l'art dramatique. L'art dramatique étant au centre du projet éducatif, l'élève est appelé à vivre une expérience scolaire différente. C'est d'ailleurs ce qui caractérise cette école et qui explique qu'elle soit choisie par plusieurs élèves éprouvant des difficultés d'apprentissage et de comportement. Cette école de la Commission Scolaire de Montréal occupe une position privilégiée dans la vie culturelle de son quartier, puisqu'elle se trouve près de nombreux théâtres et écoles de formations professionnelles de ce métier. Loin de la simple initiation à l'art dramatique, ce programme rigoureux développe un regard sensible et critique par rapport aux arts et prépare à des formations spécialisées en Arts et lettres et Communication. 
Le projet a été mis sur pied en réponse à une demande du milieu voulant se doter d'une identité propre. En 2004, la direction a donc fait appel à l'enseignante en art dramatique pour mettre sur pied ce projet particulier de formation. Depuis onze ans, les élèves travaillent les différentes techniques de jeu, la dramaturgie et la théâtralité tout en s'initiant aux nouvelles formes, notamment le multimédia.

\section{École Secondaire Thérèse-Martin}

L'École Secondaire Thérèse-Martin fait partie de la Commission Scolaire des Samares. La clientèle de cettes école provient principalement de Joliette et des villes environnantes. Implantée depuis une vingtaine d'années, la concentration en art dramatique devient en 2012, en réponse à une demande du milieu, un projet particulier de formation (arts-études). Ce programme, en plus de poursuivre la mission de l'école, développe des compétences artistiques par la mise sur pied de projet théâtral d'envergure. De ce fait, l'élève est appelé à exploiter tous les éléments du langage dramatique. La sélection se fait sur la base d'une audition et les critères reposent sur l'intérêt de l'élève pour l'art dramatique, son dossier académique, son attitude en groupe, sa capacité d'intégration et d'adaptation ainsi que sur son potentiel artistique. L'École Secondaire Thérèse-Martin se distingue par ses projets artistiques à grand déploiement. En effet, le milieu est habitué à des représentations théâtrales d'envergure ce qui a pour effet de mettre une certaine pression sur les enseignants responsables du projet.

\section{Ce qui Relie ces Pratiques Singulières}

Pour les quatre écoles que nous avons interrogées, il est intéressant de constater, outre l'enrichissement offert dans ces formations et les projets d'envergure qui s'y rattachent, l'importance accordée au développement personnel et social de l'élève. De plus, ces projets ont été proposés par le milieu (direction d'école, conseil d'établissement, regroupement de parents) notamment pour joindre un plus grand nombre d'élèves et, ainsi, rehausser la réputation de l'école. Plusieurs élèves de ces programmes poursuivent leur formation théâtrale à des niveaux supérieurs (collégiale, universitaire, conservatoire, école de théâtre). Nous remarquons également que certaines écoles acceptent les élèves sous certaines conditions, tandis que d'autres 


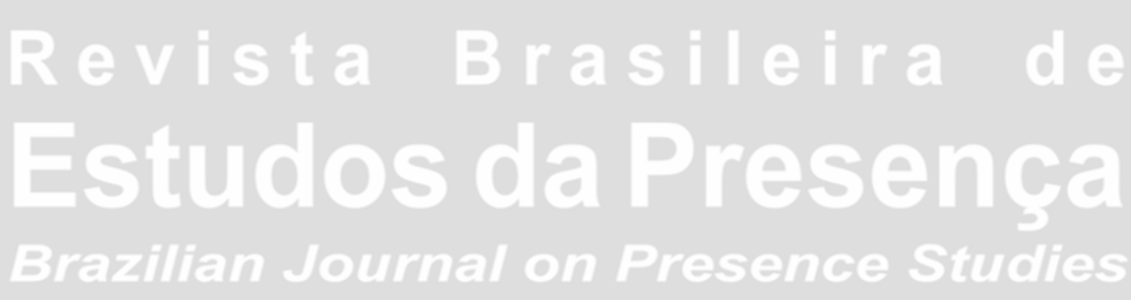

ne sélectionnent pas vraiment les élèves. Ce sont les enseignants qui ont élaboré les projets et les ont soumis, le cas échéant, au Ministère de l'Éducation, du Loisir et du Sport (MELS). La plupart offrent une formation pour les jeunes de douze à dix-sept ans, à l'exception de l'École Curé-Antoine Labelle, une école de deuxième cycle, qui accueille uniquement les élèves de quinze ans et plus. Le tableau qui suit illustre les éléments principaux reliant ces programmes de formation en art dramatique aux objectifs et retombées sur le plan personnel, dans le milieu de vie, ainsi que les obstacles rencontrés pour y parvenir. Nous y retrouvons les composantes clefs issues de l'analyse des questionnaires auxquels chacun d'entre eux a répondu.

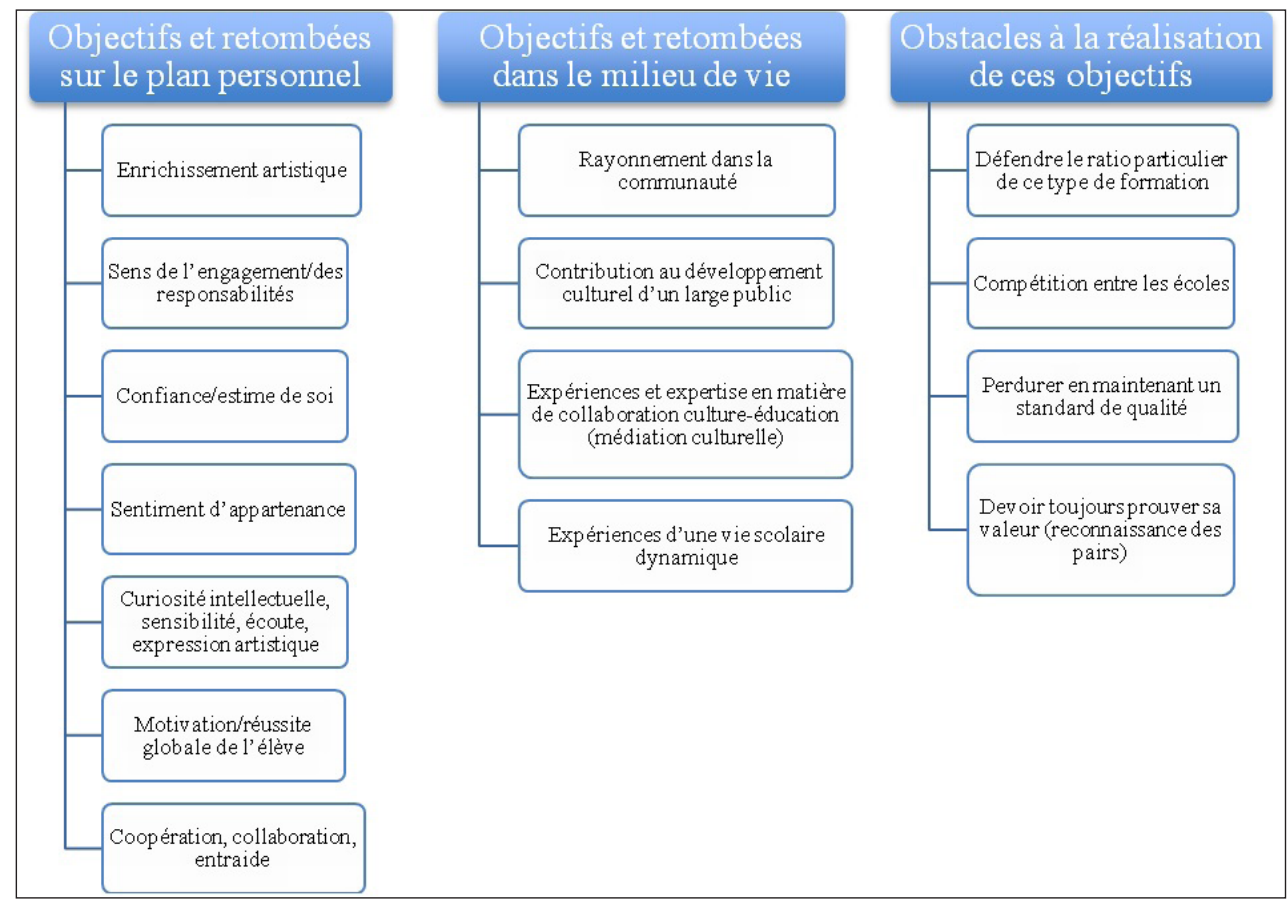

Image 5-Tableau synthèse de l'analyse des objectifs et retombées des PPF et des obstacles à leur réussite.

Cette recherche exploratoire avait pour but d'étudier certains programmes particuliers en art dramatique. A priori, nous pensions que ces programmes étaient orientés uniquement sur l'enrichissement de l'art dramatique. Cependant, bien que ces formations artistiques enrichies axent leur formation sur les différentes techniques de jeu, la dramaturgie et les techniques théâtrales et qu'elles rayonnent dans le milieu de vie, elles démontrent toutes qu'elles ont à cœur le développement personnel et social de l'élève. Bien que ces objectifs soient étroitement liés à la triple mission éducative attribuée aux écoles par la loi de l'instruction publique - qui s'articule autour de trois axes: qualifier, instruire et socialiser (Ministère de l'Éducation, du Loisir 
et du Sport, 2007) - il est intéressant de constater l'importance marquée accordée par les responsables de ces projets particuliers à la socialisation de l'élève. Ces programmes visent donc, par le biais du théâtre, la réussite scolaire par le développement de compétences disciplinaires et transversales permettant ainsi à l'élève de structurer son identité, de développer son pouvoir d'action et de se construire une vision du monde (Ministère de l'Éducation, du Loisir et du Sport, 2007). Lorsque l'on regarde les éléments qui ont été ciblés par les répondants du questionnaire, nous constatons que le développement artistique est en avant-plan, mais soutenu par un désir de développer aussi d'autres aptitudes et attitudes qui pourront être réinvesties par l'élève dans d'autres contextes ou domaines connexes.

Comme nous l'avons dit précédemment, la demande de mise sur pied de ces PPF a été faite par les directions d'école et les conseils d'établissement. Elles ont pour but d'offrir une formation différente et, ainsi, de donner une couleur particulière à l'école. Cependant, tous les répondants s'accordent pour dire qu'ils doivent constamment revendiquer le droit d'exister, particulièrement auprès des collègues de travail. Ces réponses vont dans le même sens que celles répertoriées par la FEA. On peut donc penser que les formations particulières en art dramatique n'échappent pas à la perception négative des collègues lorsque vient le temps de se répartir équitablement les tâches. En effet, une telle formation exige un ratio d'élèves plus petit, ce qui oblige les autres collègues à compenser en ayant un ratio plus grand. Bien que tous s'accordent pour reconnaitre les bienfaits de ces formations enrichies, les répondants constatent qu'ils doivent toujours prouver leur valeur afin de perdurer dans le temps tout en maintenant un standard de qualité. De plus, un des répondants a souligné la concurrence qui se crée entre les écoles d'une même commission scolaire et des attentes de plus en plus grandes qui en résultent puisque dans certaines écoles ces projets sont mis de l'avant pour attirer une plus grande clientèle. Rappelons que le syndicat (FAE) a relevé lui aussi cette retombée négative sur le milieu scolaire.

\section{L'Émergence de Voix Distinctes dans la Communauté}

L'offre de service en loisir portant sur l'art dramatique et le théâtre est assez diversifiée et touche plusieurs clientèles au Québec. Dans le cadre de cet article, nous nous intéressons plus particulièrement aux pratiques singulières, aux approches qui se distinguent soit par 
la clientèle desservie soit par le type d'activités offertes. De fait, nous retiendrons trois organismes ayant des caractéristiques communes et divergentes que nous présenterons selon les sous-critères suivants: 1) organisme fondé en réponse à une demande du milieu communautaire ou pour offrir un service; 2) objectifs; 3) clientèle et types d'activités offertes; 4) apports aux participants et retombées dans le milieu; 5) difficultés rencontrées.

\section{Les Vidanges en Cavale}

Cette compagnie de théâtre à vocation sociale a été fondée en 2008. Il s'agit d'un organisme sans but lucratif ayant pour objectif de promouvoir le potentiel créateur et l'imaginaire des jeunes et des communautés marginalisées. Elle travaille dans des contextes interculturels et offre à la fois des spectacles grand public et des formations sur mesure. Nous concentrerons notre analyse sur ce deuxième volet.

La clientèle ciblée par les formations est principalement les adolescents et les adultes provenant de milieux défavorisés, issus de l'immigration, aux prises avec des problèmes de santé mentale ou en processus de raccrochage scolaire. La compagnie offre principalement trois types de formations: Théâtre et Communication, Théâtre et Écriture Créative et Théâtre et Parcours Migratoire. Les données tirées de leur pratique pour le présent article sont principalement issues la formation de Théâtre et Communication qui s'est établie au Nunavik ainsi que dans le réseau des Carrefours Jeunesse-Emploi ${ }^{15}$ (plus spécifiquement à Laval, Montréal-Nord, Hochelaga-Maisonneuve et dans l'Ouest-de-l'île).

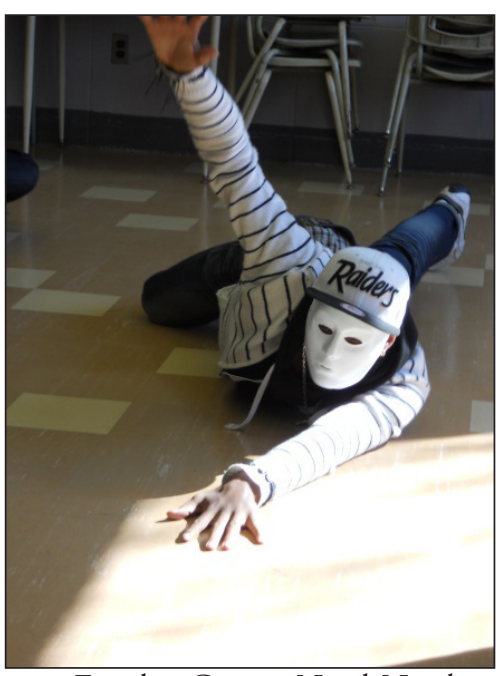

Image 6 - Carrefour Jeunesse-Emploi Centre Nord-Nord - Centre Gabrielle-Roy (2013). Photo: Marine Janssens. 


\section{Oxy-Jeunes}

Cet organisme communautaire s'est incorporé en 1985 afin de soutenir l'expression des préoccupations des jeunes par le biais des arts. Ses activités visent à contribuer à l'accomplissement des rêves des participants en développant des projets artistiques et culturels, tout en luttant contre le décrochage scolaire, la toxicomanie, la violence, la criminalité et le suicide. Les participants de douze à dix-sept ans sont autant des élèves en marge du système scolaire ayant des difficultés de développement que des jeunes sans difficulté particulière. Il est à noter, toutefois, que $65 \%$ de la clientèle est issue de l'immigration et qu'une grande partie d'entre eux ont des statuts socioéconomiques faibles. En effet, les jeunes proviennent d'un peu partout à Montréal, mais principalement du quartier où se situent les locaux d'activités, soit le quartier Centre-Sud. Les activités offertes sont donc gratuites et touchent à la création artistique pour et par les jeunes, au jumelage avec des artistes professionnels lors de projets de médiation artistique et à des sorties culturelles.

\section{Les Créateurs}

Ce programme, créé en 1992, est donné par l'organisme sans but lucratif, Gymn-O Laval (incorporé en 1978). L'organisme offre aujourd'hui aux enfants, adolescents et jeunes adultes ayant diverses difficultés d'apprentissage (TSA, trisomie, DI etc.), provenant de milieux socioéconomiques variés, des activités de loisirs adaptées à leurs besoins. Comme chaque activité offerte à Gymn-O Laval, le programme des Créateurs a vu le jour en réponse à une demande de la part des parents dont les enfants participaient à des activités déjà en place. Les Créateurs se destinent aux jeunes de onze à dix-sept ans et offrent des activités de théâtre adapté. Les participants expérimentent ce médium pendant une session de onze semaines, puis préparent un spectacle pendant une seconde session de onze semaines. Les jeunes proviennent principalement du territoire lavallois, mais comme ce type d'activité est rare, certains se déplacent depuis Montréal et sa couronne nord.

\section{Ce qui Relie ces Pratiques Singulières}

Parmi les trois organismes ciblés par cet article, l'un d'entre eux œuvre particulièrement auprès de jeunes en difficulté d'apprentissage 


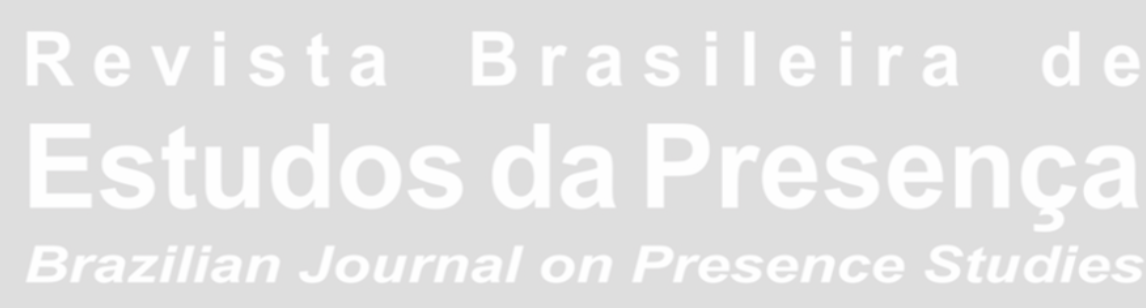

E-ISSN 2237-2660

et les deux autres travaillent auprès d'adolescents ou de jeunes adultes en marge du système scolaire. Bien que ces clientèles aient leurs caractéristiques spécifiques propres, les objectifs, leur apport aux participants, les retombées dans le milieu et les difficultés rencontrées se recoupent. En effet, chaque organisme dessert une clientèle ciblée et offre des activités d'art dramatique et de théâtre répondant aux besoins particuliers de cette dernière. Cependant, certains des objectifs et retombées peuvent être regroupés thématiquement de manière à illustrer la vocation sociale et l'approche communautaire qu'ils préconisent. Le tableau qui suit illustre les éléments principaux reliant ces organismes aux objectifs et retombées sur le plan personnel, dans le milieu de vie, ainsi que les obstacles rencontrés pour parvenir à ceux-ci. Nous y retrouvons les composantes clefs issues de l'analyse des questionnaires auxquels chacun d'entre eux a répondu.

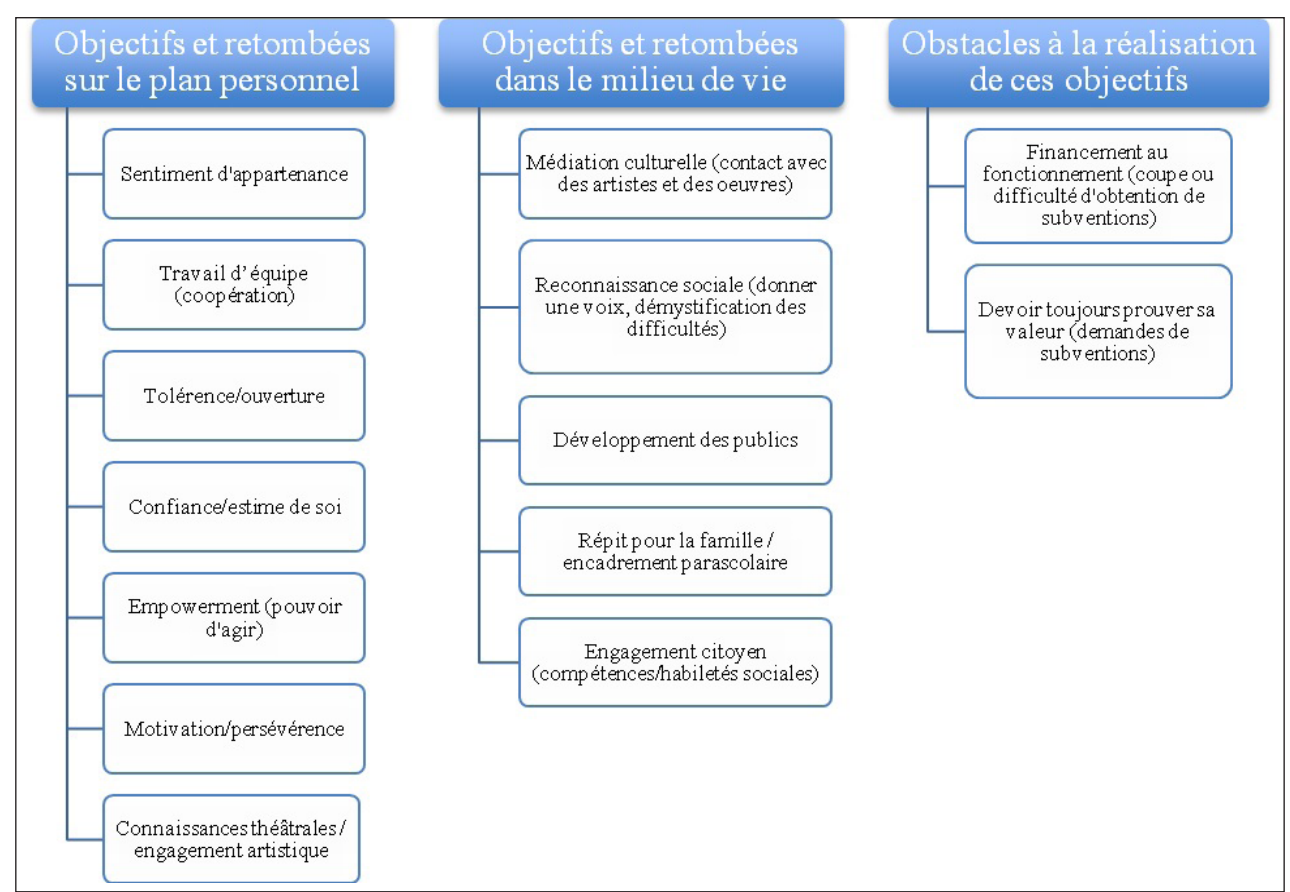

Image 7 - Tableau synthèse de l'analyse des objectifs et retombées des loisirs en théâtre et des obstacles à la réussite de ceux-ci.

Notre première hypothèse concernant les organismes communautaires (c'est-à-dire qu'ils utilisent le théâtre comme un moyen pour l'atteinte d'objectifs sur le plan du développement personnel et social des participants) est en partie confirmée. Toutefois, les résultats de cette recherche exploratoire nous ont aussi permis de voir que l'apprentissage de techniques théâtrales et de connaissances reliées aux arts de la scène était très présent. En effet, pour que les jeunes 
puissent s'exprimer efficacement par ce médium, un minimum de connaissances et d'expérience est requis. La qualité esthétique des productions est donc non-négligeable et semble contribuer aux objectifs personnels et sociaux, tels que l'estime de soi, le sentiment de réussite et la valorisation sociale de la rencontre sensible entre les participants et leur communauté. Comme nous l'avons explicité précédemment, ces organismes contribuent à un engagement social certain et favorisent le sentiment d'appartenance des participants à leur milieu. Les jeunes apprennent, en outre, des habiletés et des compétences sociales leur permettant d'être reconnus et investis comme citoyens. Considérant les objectifs poursuivis et préconisés par le MCCCF, il semble surprenant de constater la difficulté de survie financière de ces organismes. En effet, tous ont souligné la difficulté à financer leurs activités et à prouver leur nécessité.

\section{Comparaison entre le Milieu Scolaire et le Milieu Communautaire}

Notre hypothèse de départ concernant les projets particuliers de formation en art dramatique supposait que le théâtre était une fin en soi. D'autre part, nous supposions que les organismes communautaires, qui font appel au théâtre et à l'art dramatique comme moyen pour l'atteinte d'objectifs sur le plan du développement personnel et social des participants, se préoccupaient peu ou pas du résultat esthétique final.

Cependant, comme le démontre le tableau synthèse ci-dessous, à l'intérieur des deux groupes, on retrouve des objectifs et des retombées similaires. Ces éléments touchent particulièrement le développement d'attitudes nécessaires lorsqu'on désire s'engager dans un processus de création, d'interprétation ou d'appréciation en art dramatique. Que l'on soit dans une formation dans laquelle l'enrichissement artistique est au premier plan, ou que l'on soit un groupe de jeunes marginalisés désirant prendre la parole dans sa communauté, l'engagement dans la démarche artistique est fondamental. En milieu scolaire, le résultat artistique est important puisque le rayonnement dans la communauté est étroitement lié à la pérennité du programme particulier. Pour les organismes communautaires, c'est sur le plan personnel que l'on voit se manifester certains résultats leur permettant de remplir leur mandat de vocation sociale. Dans les deux contextes, malgré des objectifs divergents, des bénéfices communs émergent, au contact du théâtre et dans le processus de groupe qu'il nécessite. 


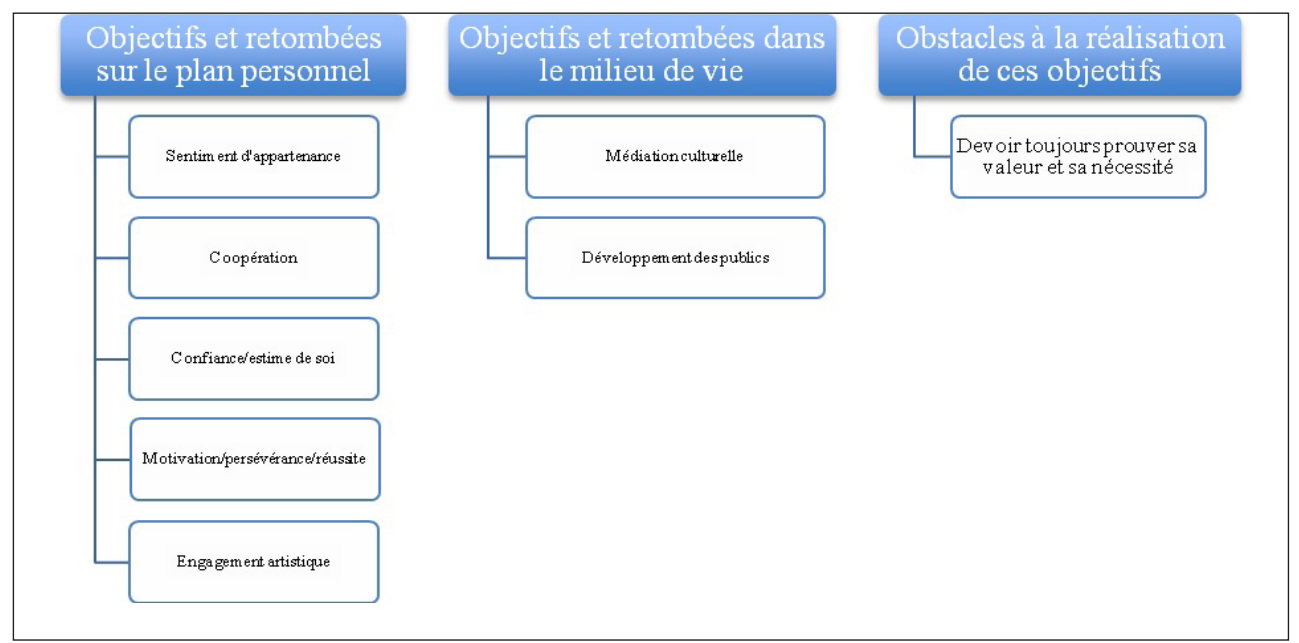

Image 8 - Tableau synthèse de l'analyse des objectifs et retombées des PPF et des loisirs en théâtre, ainsi que des obstacles à leur réussite.

Il est également intéressant de constater que dans les deux cas, les responsables de ces projets artistiques doivent constamment prouver leur valeur et leur nécessité, que ce soit auprès des pairs ou lors des demandes de subventions.

\section{Conclusion}

Les contextes scolaire et communautaire offrent des possibilités riches et variées quant à la pratique de l'art dramatique. En effet, au Québec, il existe ce que l'on appelle des projets particuliers de formation en art dans les écoles. Ceux-ci permettent à des élèves, notamment au secondaire, d'approfondir leurs connaissances théoriques et pratiques du théâtre dans le cadre d'un cheminement scolaire structurant et enrichi menant au diplôme d'études secondaires. En milieu communautaire, l'offre de service en loisir est très diversifiée et touche à une diversité de clientèles. Cependant, il existe des organismes se spécialisant en théâtre auprès des adolescents et jeunes adultes, ayant pour objectif de faire vivre aux participants des réussites contribuant à leur développement personnel et social dans le cadre d'activités de théâtre.

Bien que ces deux contextes aient pour prémisses de départ des objectifs et des clientèles différentes - élèves performant en art dramatique versus jeunes en difficultés d'apprentissage ou en marge du système scolaire - certains constats émergent de l'étude des cas multiples présentés dans cet article. Il est possible d'affirmer que les hypothèses de départ ont été confirmées: pour les projets parti- 
culiers de formation, l'art dramatique est une fin en soi, alors que pour les organismes communautaires, l'art dramatique est un moyen pour l'atteinte d'objectifs sur le plan du développement personnel et social des participants. Un troisième constat nous est apparu en cours d'analyse concernant des bienfaits communs émergeant de la pratique théâtrale. De fait, par un engagement dans un processus de création menant à la production d'un spectacle ou lors d'exercices d'exploration, les jeunes vivent une expérience de groupe rassembleuse et enrichissante sur le plan personnel. Plus encore, dans les deux cas, la nécessité de s'approprier les techniques théâtrales pour arriver à s'exprimer esthétiquement et rejoindre son auditoire de manière convaincante et sensible est de mise.

Mentionnons que les résultats concernant les obstacles liés à l'atteinte des objectifs des organismes communautaires et des projets particuliers de formation en art dramatique se recoupent. Nous pouvons constater que, dans les deux cas, la nécessité de prouver la valeur, la pertinence, voire la nécessité de ces pratiques singulières semble constante et à refaire régulièrement, et ce même si la demande de tels projets provient du milieu. Qu'il s'agisse de demandes de subventions au fonctionnement ou le maintien dans la grille horaire des options offertes par l'école, l'exercice nécessite un effort continuel de renouvèlement et de démonstration des bénéfices aux participants, au milieu de vie, à la communauté où les programmes se logent, ainsi qu’aux subventionnaires, le cas échéant.

Conscientes de la charge de travail que cela représente pour les enseignants, les intervenants et les artisans du milieu scolaire et communautaire de coordonner et de mener à bien ces programmes particuliers (fonctionnement, durabilité, efficacité et qualité), il semble tout à fait indiqué de déléguer ce travail à d'autres qui pourraient soutenir cette démarche avec crédibilité. Qui plus est, la nécessité d'avoir une certaine distance pour porter un regard critique sur les différentes approches artistiques et pédagogiques mises en ouvre confirme cette option.

En ce sens, il nous semble apparent qu'un partenariat entre les milieux de pratique et de recherche soit une voie à privilégier. En effet, la recherche peut avoir pour objectif de faire ressortir, de manière systématique, les retombées et les impacts des pratiques dans leur milieu. En contrepartie, la possibilité d'étudier de près des for- 
mations particulières en art dramatique permettrait aux chercheurs de contribuer aux connaissances actuelles dans le domaine. Les résultats, issus de ces études, pourraient s'inscrire dans la formation des maîtres (en donnant un portrait plus juste de ce qui se fait dans les écoles et dans le milieu communautaire) et ainsi de faire valoir leur importance plus largement que dans les milieux de pratiques, c'est-à-dire nationalement et internationalement.

Dans ce cas-ci, tel n'était pas notre objectif: nous voulions plutôt voir comment les PPF et organismes s'expliquent eux-mêmes leur réussite et leur nécessité d'exister. Le but étant de dresser un premier portrait de la situation, nous n'avons pas cherché à analyser les raisons/ causes/facteurs qui expliquent cette nécessité. Conséquemment, dans un deuxième temps, une analyse rigoureuse et théorique basée sur les mécanismes à l'œuvre (parfois sans doute à l'insu même des écoles/ enseignants/directions et des organisateurs/animateurs communautaires) dans leur travail effectué auprès des jeunes pourrait être faite.

Enfin, cette recherche exploratoire nous a permis de poser les premiers jalons d'une recherche à plus grande échelle qui pourra nous donner l'occasion de dresser un portrait plus juste des pratiques singulières au Québec et d'aller au-delà de nos conclusions actuelles, qui bien qu'elle donne un premier aperçu de la réalité québécoise, nous informe peu sur les procédés à l'œuvre qui en font des pratiques exemplaires. Nous espérons donc pouvoir poursuivre notre étude avec un échantillonnage plus grand nous offrant la possibilité de mieux cerner les causes et caractéristiques qui définissent les réalités diverses de ces pratiques partout au Québec. Quels sont les impacts de l'éloignement, de la composition et de la densité de la population d'une région, de la médiation culturelle et l'accessibilité à la culture, sur les pratiques singulières de l'art dramatique en milieu scolaire et communautaire? Certes, répondre à ces questions permettrait d'explorer et de comprendre comment l'offre culturelle de loisir et la formation particulière en art dramatique sont pratiquées au Québec et de contribuer à une meilleure connaissance concernant les tenants et aboutissants de ces pratiques singulières. 


\section{Notes}

${ }^{1}$ Pour avoir accès à la forme dynamique du questionnaire, veuillez contacter les chercheures: Carole Marceau (marceau.carole@uqam.ca) et Maud Gendron-Langevin (gendron-langevin. maud@uqam.ca).

${ }^{2}$ Les participants interrogés au moyen des questionnaires sont les responsables de projets particuliers de formation dans le cadre scolaire et les coordonnateurs de projets artistiques ou la direction artistique dans le milieu communautaire.

${ }^{3}$ En ce qui concerne les programmes en arts, il s'agit de la troisième mouture de programme de formation pour l'enseignement de l'art dramatique.

${ }^{4}$ Le conseil d'établissement est formé d'au moins quatre parents, quatre membres du personnel - dont au moins deux enseignants - de deux représentants de la communauté, ainsi que de deux élèves du secondaire et d'un membre du personnel des services de garde, le cas échéant.

${ }^{5}$ L'enseignant titulaire d'une classe au primaire détient un baccalauréat pour enseigner les matières de base au primaire et au préscolaire.

${ }^{6}$ Le spécialiste détient un baccalauréat spécialisé en enseignement d'une discipline artistique pour le primaire et le secondaire.

${ }^{7}$ Les Projets Pédagogiques Particuliers (PPP) ne sont pas créés uniquement en arts, on dénombre plusieurs projets de sports-études et de langues-études ainsi que les programmes d'éducation internationale (PEI). Bien que certaines de nos données concernent l'ensemble des PPP, nous traiterons particulièrement de ceux en art dramatique.

${ }^{8}$ Ce sont les articles 222, 240 et 447.2 de cette loi qui s’appliquent (Fédération Autonome de l'Enseignement, 2011-2012, n.p.).

${ }^{9}$ L'un des deux syndicats des enseignants primaire/secondaire.

${ }^{10}$ Les résultats présentés concernent l'ensemble des municipalités du Québec, bien que notre recherche porte plus spécifiquement sur la grande région métropolitaine de Montréal.

${ }^{11}$ L'échantillon comptait 6.878 répondants âgés de quinze ans et plus dans dix-sept régions administratives du Québec.

12 Par amateur, nous entendons la pratique artistique non rémunérée, par des non professionnels (n'ayant reçu aucune formation artistique professionnelle reconnue).

13 «[...] La pratique libre fait référence à la pratique d'une activité artistique ou culturelle en amateur, la pratique organisée est associée au fait de suivre un cours ou un atelier d'art [...]» (Survol, 2012, p. 16).

${ }^{14}$ Donnée provenant du site officiel du Ministère de l'Éducation des loisirs et du sport.

${ }^{15}$ Les carrefours jeunesse-emploi ont comme mandat d'accompagner et de guider les jeunes adultes de seize à trente-cinq ans dans leurs démarches d'insertion sociale et économique. Les services offerts les aident dans leur cheminement vers l'emploi, vers un retour aux études ou dans le démarrage d'une petite entreprise. 


\section{Références}

ASSOCIATION QUÉBÉCOISE DU LOISIR MUNICIPAL. Le Loisir dans les Nouvelles Villes: les clientèles particulières en loisir dans un contexte de fusion municipale. Québec, 2002. Disponible sur: <http://www.loisirmunicipal.qc.ca>. Consulté le: 11 juin 2014.

FÉDÉRATION AUTONOME DE L'ENSEIGNEMENT. Les Retombées dans le Milieu Scolaire selon la Fédération Autonome de l'Enseignement. Québec, 2011-2012. Disponible sur: <http://www.lafae.qc.ca>. Consulté le: 20 juin 2014.

FÉdÉRATION DES COMITÉS DE PARENTS DU QUÉBEC. Le Conseil d'Établissement au Centre de l'École. Québec, 2009. Disponible sur: <http://www.fcpq. qc.ca>. Consulté le: 23 juin 2014.

FÉDÉRATION QUÉBÉCOISE DES CENTRES COMMUNAUTAIRES. Cadre de Référence des Centres Communautaires de Loisirs Fédérés: approches et perspectives. Québec, 2013. Disponible sur: <http://www.fqccl.org>. Consulté le: 11 juin 2014.

GOUVERNEMENT DU QUÉBEC. La Culture, toute une École! Québec, 2013. Disponible sur: <http://www.mels.gouv.qc.ca>. Consulté le: 29 juin 2014.

GOUVERNEMENT DU QUÉBEC. Plan Stratégique 2009-2013 du Ministère de l'Éducation, du Loisir et du Sport. Québec, 2009. Disponible sur: <http://www.mels. gouv.qc.ca>. Consulté le: 18 juin 2014.

GOUVERNEMENT DU QUÉBEC. La Politique Culturelle du Québec: notre culture, notre avenir. Québec, 1996. Disponible sur: <http://www.mcc.gouv.qc.ca>. Consulté le: 11 juin 2014.

GOUVERNEMENT DU QUÉBEC. Programme de Formation de l'École Québécoise: enseignement secondaire, deuxième cycle. Québec: Ministère de l'Éducation, du Loisir et du Sport, 2007. Disponible sur: <http://wwwl.mels.gouv.qc.ca>. Consulté le: 15 juin 2014. GOUVERNEMENT DU QUÉBEC. Projets Particuliers en Arts. Québec. Disponible sur: <http://www.mels.gouv.qc.ca>. Consulté le: 13 juil. 2014.

GOUVERNEMENT DU QUÉBEC. Vivre avec une Incapacité au Québec: un portrait statistique à partir de l'enquête sur la participation et les limitations d'activités de 2001 et 2006. Québec: Institut de la Statistique du Québec, 2010. Disponible sur: <http://www. stat.gouv.qc.ca>. Consulté le: 11 juin 2014.

MINISTÈRE DE LA CULTURE ET DES COMMUNICATIONS. Rôle du Ministère: le loisir, une dimension essentielle pour la culture. Québec, 2013. Disponible sur: <http:// www.mcc.gouv.qc.ca>. Consulté le: 16 juin 2014.

SURVOL: bulletin de la recherche et de la statistique. Québec: Gouvernement du Québec/ Ministère de la Culture, des Communications et de la Condition Féminine, n. 22, jun. 2012. Disponible sur: <https://www.mcc.gouv.qc.ca/fileadmin/documents/publications/ Survol-22-juin2012.pdf>. Consulté le: 16 juin 2014.

UNE ÉCOLE MONTRÉAlAISE POUR TOUS. À Propos de Nous. Montréal, 2011. Disponible sur: <http://www.ecolemontrealaise.info>. Consulté le: 29 juin 2014. 
Carole Marceau est professeur en enseignement de l'art dramatique à l'Université du Québec à Montréal (UQAM). Elle s'intéresse au processus de création, à l'évaluation des apprentissages artistiques et à la pratique pédagogique dans une classe d'art. Elle est corédactrice de deux revues portant sur l'enseignement de l'art dramatique.

E-mail: marceau.carole@uqam.ca

Maud Gendron-Langevin est professeur en enseignement de l'art dramatique l'Université du Québec à Montréal (UQAM), chercheuse et membre du pôle de recherche Arts, Créativité et Santé. Elle s'intéresse à l'intervention par le biais du théâtre auprès de jeunes ayant des besoins particuliers.

E-mail: gendron-langevin.maud@uqam.ca

Ce texte inédit, révisé par Annelyse Gayraud, est également publié en portugais dans ce numéro. 